\title{
Schizophrenia risk gene CAV1 is both pro-psychotic and required for atypical antipsychotic drug actions in vivo
}

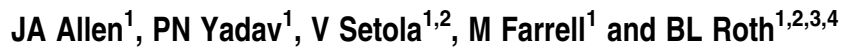

Caveolin-1 (Cav-1) is a scaffolding protein important for regulating receptor signaling cascades by partitioning signaling molecules into membrane microdomains. Disruption of the CAV1 gene has recently been identified as a rare structural variant associated with schizophrenia. Although Cav-1 knockout (KO) mice displayed no baseline behavioral disruptions, Cav-1 KO mice, similar to schizophrenic individuals, exhibited increased sensitivity to the psychotomimetic $\mathrm{N}$-methyl-D-aspartate receptor antagonist phencyclidine (PCP). Thus, PCP disruption of prepulse inhibition (PPI) and PCP-induced mouse locomotor activity were both enhanced by genetic deletion of Cav-1. Interestingly, genetic deletion of Cav-1 rendered the atypical antipsychotics clozapine and olanzapine and the 5- $\mathrm{HT}_{2 \mathrm{~A}}$-selective antagonist $\mathrm{M} 100907$ ineffective at normalizing PCP-induced disruption of PPI. We also discovered that genetic deletion of Cav-1 attenuated $5-\mathrm{HT}_{2 \mathrm{~A}}$-induced $\mathrm{c}-\mathrm{Fos}$ and egr-1 expression in mouse frontal cortex and also reduced $5-\mathrm{HT}_{2 \mathrm{~A}}$-mediated $\mathrm{Ca}^{2}{ }^{2}$ mobilization in primary cortical neuronal cultures. The behavioral effects of the $5-\mathrm{HT}_{2 \mathrm{~A}}$ agonist (2,5-dimethoxy-4-iodoamphetamine) including head twitch responses and disruption of PPI were also attenuated by genetic deletion of Cav-1, indicating that Cav-1 is required for both inverse agonist (that is, atypical antipsychotic drug) and agonist actions at $5-\mathrm{HT}_{2 \mathrm{~A}}$ receptors. This study demonstrates that disruption of the CAV1 gene-a rare structural variant associated with schizophrenia-is not only pro-psychotic but also attenuates atypical antipsychotic drug actions.

Translational Psychiatry (2011) 1, e33; doi:10.1038/tp.2011.35; published online 16 August 2011

\section{Introduction}

Schizophrenia-affecting $1 \%$ of the world's population-is a genetically heterogeneous disease with devastating consequences to affected individuals. ${ }^{1-4}$ It now appears that schizophrenia is due to both common and rare gene mutations as well as copy-number variations. The identification and study of exceedingly rare mutations such as DISC1 has had a major impact on our understanding of the potential genetic underpinnings of psychiatric disease. ${ }^{5,6}$ Recent analyses of genomic structural variations in individuals with schizophrenia indicated that the gene caveolin-1 (CAV1) is disrupted by an insertional mutation, thereby identifying CAV1 as a rare structural variant associated with schizophrenia. ${ }^{7}$

The CAV1, -2 and -3 genes encode caveolins, which represent a family of multi-functional scaffolding proteins and cholesterol-binding proteins that mediate cholesterol trafficking to lipid microdomains at the plasma membrane. ${ }^{8}$ Caveolins also function as protein scaffolds to organize proteins and lipids together in membrane domains and thereby control various cellular functions including vesicular trafficking, lipid homeostasis and signal transduction., ${ }^{9,10}$ Cav-1 and Cav-2 are widely expressed in the central and peripheral nervous systems mainly in cortical and hippocampal neurons as well as brain microvessels, astrocytes, oligodendrocytes, Schwann cells and dorsal root ganglia cells ${ }^{10-12}$ while Cav-3 is muscle-specific. Transcriptional profiling studies of mRNAs demonstrate that both Cav-1 and Cav-2 are expressed in relevant central nervous system cells including layer III and V pyramidal cortical neurons. ${ }^{13}$

The relevant functional role(s) of Cav-1 vis-a-vis the pathogenesis of schizophrenia is undefined. As many $G$ protein-coupled neurotransmitter receptors, heterotrimeric $G$ proteins and signaling effectors are known Cav-1 interacting partners, ${ }^{10,11,14}$ disruption of $C A V 1$ will likely impair neuronal signaling thereby leading to symptoms of schizophrenia in susceptible individuals. Targeted disruption of the CAV1 gene in mice provides a knockout (KO) animal model, which recapitulates the life-long disruption of CAV1 seen as a rare structural variant associated with schizophrenia. Here, we report that genetic deletion of Cav-1 in mice induces a propsychotic-like condition whereby mice are supersensitive to the psychotomimetic actions of the $N$-methyl-D-aspartate (NMDA)-receptor antagonist phencyclidine (PCP). Furthermore, Cav-1 KO mice are also resistant to the actions of atypical antipsychotic drugs and selected serotonergic agents implying an underlying disruption of serotonergic signaling also is present. These findings suggest the disruption in CAV1

\footnotetext{
${ }^{1}$ Department of Pharmacology, University of North Carolina, Schools of Medicine and Pharmacy, Chapel Hill, NC, USA; ${ }^{2}$ National Institute of Mental Health Psychoactive Drug Screening Program, University of North Carolina, Schools of Medicine and Pharmacy, Chapel Hill, NC, USA; ${ }^{3}$ Department of Psychiatry, University of North Carolina, Schools of Medicine and Pharmacy, Chapel Hill, NC, USA and ${ }^{4}$ Department of Medicinal Chemistry and Natural Products, University of North Carolina, Schools of Medicine and Pharmacy, Chapel Hill, NC, USA

Correspondence: Professor BL Roth, Department of Pharmacology, University of North Carolina, Schools of Medicine and Pharmacy, 4007 Genetic Medicine Building, Chapel Hill, NC 27599-7365, USA.
}

E-mail: bryan_roth@med.unc.edu

Keywords: antipsychotic; caveolin; clozapine; 5-HT2A; schizophrenia

Received 11 May 2011; accepted 7 July 2011 
arising in human schizophrenia could lead to treatment nonresponsiveness to atypical antipsychotics because of intrinsic alterations in serotonergic signaling.

\section{Materials and methods}

See Supplementary Information for additional materials and methods.

In vivo studies in mice. All experiments were approved by the Institutional Animal Care and Use Committees at the University of North Carolina, Chapel Hill, NC, USA. Cav-1 KO mice were obtained from the Jackson Laboratories (Bar Harbor, ME, USA) and were housed under standard conditions-12-h light-dark cycle with food and water provided ad libitum. Adult, age-matched (10-15 week) male and female littermate wild-type and Cav-1 KO drug-naive mice were used for all behavioral testing.

Prepulse inhibition. Prepulse inhibition (PPI) testing and analysis was performed as described previously. ${ }^{15,16}$ Adult male and female littermate wild-type and Cav-1 KO mice were pre-administered $\left(10 \mathrm{ml} \mathrm{kg}^{-1}\right.$, intraperitoneally (i.p.)) vehicle $(0.9 \%$ saline; $50 \mathrm{~mm}$ tartaric acid), clozapine $(0.5$, $\left.1.0 \mathrm{mg} \mathrm{kg}^{-1}\right)$, olanzapine $\left(0.5 \mathrm{mg} \mathrm{kg}^{-1}\right)$ or M100907 (0.5, $1.0 \mathrm{mg} \mathrm{kg}^{-1}$ ) and returned to their home cage. After $30 \mathrm{~min}$, animals were administered phencyclindine $(2.0,4.0$ or $6 \mathrm{mg} \mathrm{kg}^{-1}$, i.p.) and immediately placed into the PPI apparatus (SR-LAB Startle Response System, San Diego Instruments, San Diego, CA, USA). The auditory pulse protocol, test trials used and PPI calculation have been reported previously. ${ }^{15,16}$ The data were analyzed with SR$\mathrm{LAB}$ programs and are presented as means \pm s.e.m. The calculated PPI values from all groups were analyzed using a one-way analysis of variance followed by Newman-Keuls multiple comparison test with Graphpad Prism 5.0 (Graphpad Software Inc., La Jolla, CA, USA). In all cases, $P<0.05$ was considered statistically significant.

Open field activity. Locomotor activity was assessed under standardized environmental conditions in $25.8 \times 25.8 \mathrm{~cm}$ Plexiglas chambers with photo-beams spaced at $1.52 \mathrm{~cm}$ (AccuScan Instruments, Columbus, OH, USA) as previously described. ${ }^{15,16}$ For dose responses to PCP, mice were placed into the open field and $30 \mathrm{~min}$ later, PCP $\left(2.0,4.06 .0 \mathrm{mg} \mathrm{kg}^{-1}\right)$ was administered and animals were immediately returned to the open field for $90 \mathrm{~min}$. For antipsychotic studies, mice were injected (i.p.) with vehicle $(0.9 \%$ saline, $50 \mathrm{~mm}$ tartaric acid), clozapine $\left(1.0 \mathrm{mg} \mathrm{kg}^{-1}\right), \mathrm{M} 100907\left(0.1,1.0 \mathrm{mg} \mathrm{kg}^{-1}\right)$ or haloperidol $\left(0.1 \mathrm{mg} \mathrm{kg}^{-1}\right)$ and placed into the open field. After $30 \mathrm{~min}, \mathrm{PCP}\left(6.0 \mathrm{mg} \mathrm{kg}^{-1}\right)$ was administered and mice were immediately returned to the open field for $90 \mathrm{~min}$. Activity was monitored throughout this entire period. Horizontal activity was measured as the total distance traveled in centimeters. Stereotypy counts reported correspond to the number of times that stereotypic behavior was observed in the animal. A break in stereotypy of $1 \mathrm{~s}$ or more was required to separate one stereotypic episode from the next. If the animal breaks the same beam (or set of beams) repeatedly, the monitor considers that the animal is exhibiting stereotypy and each event is counted. The means \pm s.e.m. of the locomotor responses and stereotypy counts were analyzed using Graphpad Prism 5.0. Cumulative responses were analyzed using a one-way analysis of variance followed by Newman-Keuls multiple comparison test and statistical significant was set at $P<0.05$.

DOl-induced head twitch. Wild-type (WT) and Cav-1 KO littermate mice were injected i.p. with saline, or 0.25 to $1.25 \mathrm{mg} \mathrm{kg}^{-1}$ of DOI (2,5-dimethoxy-4-iodoamphetamine). The number of head twitches was counted and recorded in $5 \mathrm{~min}$ bins for a 35-min period immediately after injection. A subset of the 1.0 and $1.25 \mathrm{mg} \mathrm{kg}^{-1}$ injections were counted by two observers, one of whom was blinded to the genotype; similar results were obtained by both observers (data not shown). All the other head twitch experiments were performed by one blinded observer. The means \pm s.e.m. of the head twitch responses were analyzed using Graphpad Prism 5.0 and statistical differences analyzed using a one-way analysis of variance followed by Newman-Keuls multiple comparison test and statistical significant was set at $P<0.05$.

Quantitative reverse transcriptase-PCR for c-FOS and egr-1 mRNA expression. WT and Cav-1 KO littermates were injected with the $5-\mathrm{HT}_{2}$-selective agonist $\mathrm{DOI}$ (1.0 $\mathrm{mg} \mathrm{kg}^{-1}$, i.p.) and after $1 \mathrm{~h}$, mice were euthanized, and total RNA was immediately isolated from micro-dissected frontal cortex tissue using Trizol (Invitrogen, Carlsbad, CA, USA). In all, $10 \mu \mathrm{g}$ of RNA was treated with DNAse (DNA-free, Ambion, Austin, TX, USA), and first strand complementary DNA was synthesized from $2 \mu \mathrm{g}$ of the DNase-treated RNA using the Superscript III RNase H Reverse Transcriptase kit (Invitrogen), with Oligo-(dT) primers (Invitrogen). Mouse cFos and egr-1 complementary DNA was amplified using Power SYBR Green PCR master mix (Applied Biosystems, Invitrogen, Carlsbad, CA, USA) in the 7500 Real-time PCR System (Applied Biosystems). The relative amount of c-Fos or egr-1-specific mRNA was determined by normalizing with $\beta$-actin mRNA present in the same samples and is expressed as fold above the saline-treated littermate WT, heterozygous (HET) or Cav-1 KO samples. The following gene-specific primers were used in PCR-mouse C-Fos: sense 5'-CAA CACACAGGACTTTTGCG-3', anti-sense 5'-GGAGATAGCT GCTCTACTTTG-3'; mouse $\beta$-actin: sense 5'-TGTTACCAA CTGGGACGACA-3'; anti-sense 5'-CTGGGTCATCTTTTCA CGGT-3'. Mouse egr-1: sense 5'-TCCTCTCCATCACATG CCTG-3'; anti-sense 5'-CACTCTGACACATGCTCCAG-3'. Statistical comparisons of the means \pm s.e.m. of the transcript levels were analyzed using Graphpad Prism 5.0 using a one-way analysis of variance followed by Newman-Keuls multiple comparison test.

\section{Results}

Cav-1 KO mice exhibit increased sensitivity to the behavioral effects of the psychotomimetic PCP. PCP is a psychotomimetic NMDA glutamate receptor antagonist and PCP administration models in rodents various behavioral disruptions similar to those seen in humans with 
schizophrenia. ${ }^{17,18}$ Additionally, in non-psychotic individuals, PCP induces psychosis, cognitive disruptions and negative symptoms similar to those observed in schizophrenia thus providing evidence for the NMDA hypofunction hypothesis of schizophrenia. ${ }^{19}$ Notably, administration of PCP or its analog ketamine to individuals with schizophrenia greatly exacerbates preexisting symptoms. ${ }^{20,21}$ Accordingly, we investigated the effects of PCP in WT and Cav-1 KO mice. Cav-1 KO mice were generated previously by targeted disruption of exons 1 and 2 in the CAV1 gene by insertion of a neomycin cassette using homologous recombination ${ }^{22}$ thereby recapitulating the insertional deletion observed as a rare variant in schizophrenia.

PCP disruption of PPI is a well-established pharmacological approach to model the disrupted sensory motor gating seen in schizophrenia and related disorders. ${ }^{18,23}$ Cav-1 KO mice exhibited an enhanced response to PCP compared with WT mice (Figure 1a). At all prepulse levels tested, $4 \mathrm{mg} \mathrm{kg}^{-1} \mathrm{PCP}$ significantly disrupted PPI in Cav-1 KO mice but not in WT mice. At a higher dose of $6 \mathrm{mg} \mathrm{kg}^{-1}, \mathrm{PCP}$ significantly disrupted PPI in both genotypes. PCP disrupted PPI in Cav$1 \mathrm{KO}$ mice to a larger extent than in WT mice, particularly at the 4 and $8 \mathrm{~dB}$ prepulse levels. In behavioral phenotyping control studies, WT and Cav-1 KO mice exhibited similar startle intensities to all auditory stimuli tested (Supplementary Figure S1a) and no significant difference in baseline PPI was determined between genotypes (Supplementary Figure S1b).

Next, we examined the effect of PCP on mouse locomotor activity in the open field locomotion test. Before drug administration mice were acclimated to the novel environment for $30 \mathrm{~min}$; the novelty-induced locomotion during this initial 30-min period was similar in WT and Cav-1 KO mice (Figure 1b). Similar results were observed when mice were examined for novelty-induced locomotion over a 60-min period (Supplementary Figures S1c and d). Strikingly, administration of $4 \mathrm{mg} \mathrm{kg}^{-1} \mathrm{PCP}$ significantly increased locomotor activity in Cav-1 KO mice in comparison with WT mice (Figures $1 \mathrm{~b}$ and $\mathrm{c}$ ). In dose response studies, Cav-1 KO mice displayed increased total distance traveled in response to 4 and $6 \mathrm{mg} \mathrm{kg}^{-1}$ PCP compared with WT mice. In addition, we also discovered that Cav-1 KO mice showed an increased number of stereotypic episodes in response to PCP compared with WT mice (Figures 1d and e). In the PCP dose response studies, the stereotypy counts (measured as repetitive beam breaks) in response to 4 and $6 \mathrm{mg} \mathrm{kg}^{-1} \mathrm{PCP}$ was significantly increased in Cav-1 KO mice. Collectively, these findings indicate genetic loss of Cav-1 sensitizes mice to the effects of $\mathrm{PCP}$ in a manner reminiscent of the enhanced sensitivity individuals with schizophrenia display to PCP's psychotomimetic actions.

CAV1 KO mice show reduced sensitivity to atypical but not typical antipsychotic drugs. Cav-1 is a scaffold for both $\mathrm{D}_{2}$-dopamine and $5-\mathrm{HT}_{2 \mathrm{~A}}$ serotonin receptors-both of which represent canonical targets for typical (for example, $D_{2}$ ) and most atypical (for example, 5- $-\mathrm{T}_{2 \mathrm{~A}}$ and $\mathrm{D}_{2}$ ) antipsychotic drugs. ${ }^{14,24-26}$ Therefore, we assessed antipsychotic drug activity in WT and Cav-1 KO littermate mice. As can be seen in Figure 2a, clozapine normalized the PCP-induced disruption of PPI in WT mice at all prepulse levels and doses tested. In contrast, clozapine was ineffective at normalizing PCP disrupted PPI in Cav-1 KO mice at the 4 and $8 \mathrm{~dB}$ prepulse levels. To further confirm this phenomenon of attenuated atypical antipsychotic activity, mice were also administered the structurally related drug olanzapine. As observed with clozapine, olanzapine was also ineffective at normalizing PCP disrupted PPI in Cav-1 KO mice (Supplementary Figure S2). To determine if this altered antipsychotic activity in Cav-1 KO mice is due to altered serotonin signaling, mice were administered the $5-\mathrm{HT}_{2 \mathrm{~A}^{-}}$ selective antagonist M100907, which is known to reverse PCP-induced disruption of PPI. ${ }^{15}$ Importantly, M100907 was also ineffective at normalizing PCP disrupted PPI in Cav-1 $\mathrm{KO}$ mice, particularly at the 4 and $8 \mathrm{~dB}$ prepulse levels (Figure 2b). The observation that M100907 is ineffective in Cav-1 KO mice suggests antagonism of $5-\mathrm{HT}_{2 \mathrm{~A}}$ receptors is ineffective in these mice; ineffective $5-\mathrm{HT}_{2 \mathrm{~A}}$ antagonism because of loss of Cav-1 also likely explains the ineffectiveness of clozapine and olanzapine in this behavioral index of atypical antipsychotic drug action.

In a complementary behavioral assessment, we examined the effects of these medications to normalize PCP-induced hyperlocomotion. WT and Cav-1 KO mice were administered vehicle followed by PCP, clozapine followed by PCP, or haloperidol followed by PCP and locomotor activity was measured. Clozapine significantly decreased PCP-induced locomotion and total distance traveled in WT but not Cav-1 KO mice (Figures $2 c$ and d), further indicating clozapine efficacy is decreased in Cav-1 KO mice.

Interestingly, the $\mathrm{D}_{2}$-dopamine receptor antagonist and typical antipsychotic haloperidol was equally effective in both genotypes and significantly decreased PCP-induced locomotion (Figures $2 \mathrm{c}$ and $\mathrm{d}$ ). This effectiveness of haloperidol in both genotypes indicates antagonism of $D_{2}$-dopamine receptors is preserved in Cav-1 KO mice. This also suggests the attenuated clozapine and olanzapine effects observed in Cav-1 KO mice are not due to alterations in $D_{2}$ receptor antagonist actions. Antagonism of $5-\mathrm{HT}_{2 \mathrm{~A}}$ receptors by M100907 provided additional insights. WT and Cav-1 mice were administered vehicle or M100907 followed by PCP and locomotor activity was determined as before. M100907 significantly decreased PCP-induced locomotion in WT and Cav-1 KO mice; however, M100907 was less effective in Cav-1 KO mice compared with WT mice (Figures $2 e$ and f). Together, these results indicate genetic deletion of $\mathrm{Cav}-1$ attenuates atypical antipsychotic and $5-\mathrm{HT}_{2 \mathrm{~A}}$ antagonist efficacy and that Cav-1 is required for the actions of these drugs to normalize the psychotomimetic-like effects of PCP in mice.

Mouse behavioral and biochemical responses to $5-\mathrm{HT}_{2 \mathrm{~A}}$ receptors are attenuated by genetic deletion of CAV1. The observation that both atypical antipsychotic drugs and $5-\mathrm{HT}_{2 \mathrm{~A}}$ receptor inverse agonists are ineffective in Cav-1 KO mice prompted us to examine $5-\mathrm{HT}_{2 \mathrm{~A}}$ agonist related behaviors and signaling responses. One reliable and extensively validated behavioral readout for $5-\mathrm{HT}_{2 \mathrm{~A}}$ agonism is the head twitch response. ${ }^{16,27,28}$ Thus, littermate WT and Cav-1 KO mice were administered $1.25 \mathrm{mg} \mathrm{kg}^{-1} \mathrm{DOI}$ and head twitches scored over a 35-min period; DOI-induced head twitches were attenuated in Cav-1 KO mice at all-time 

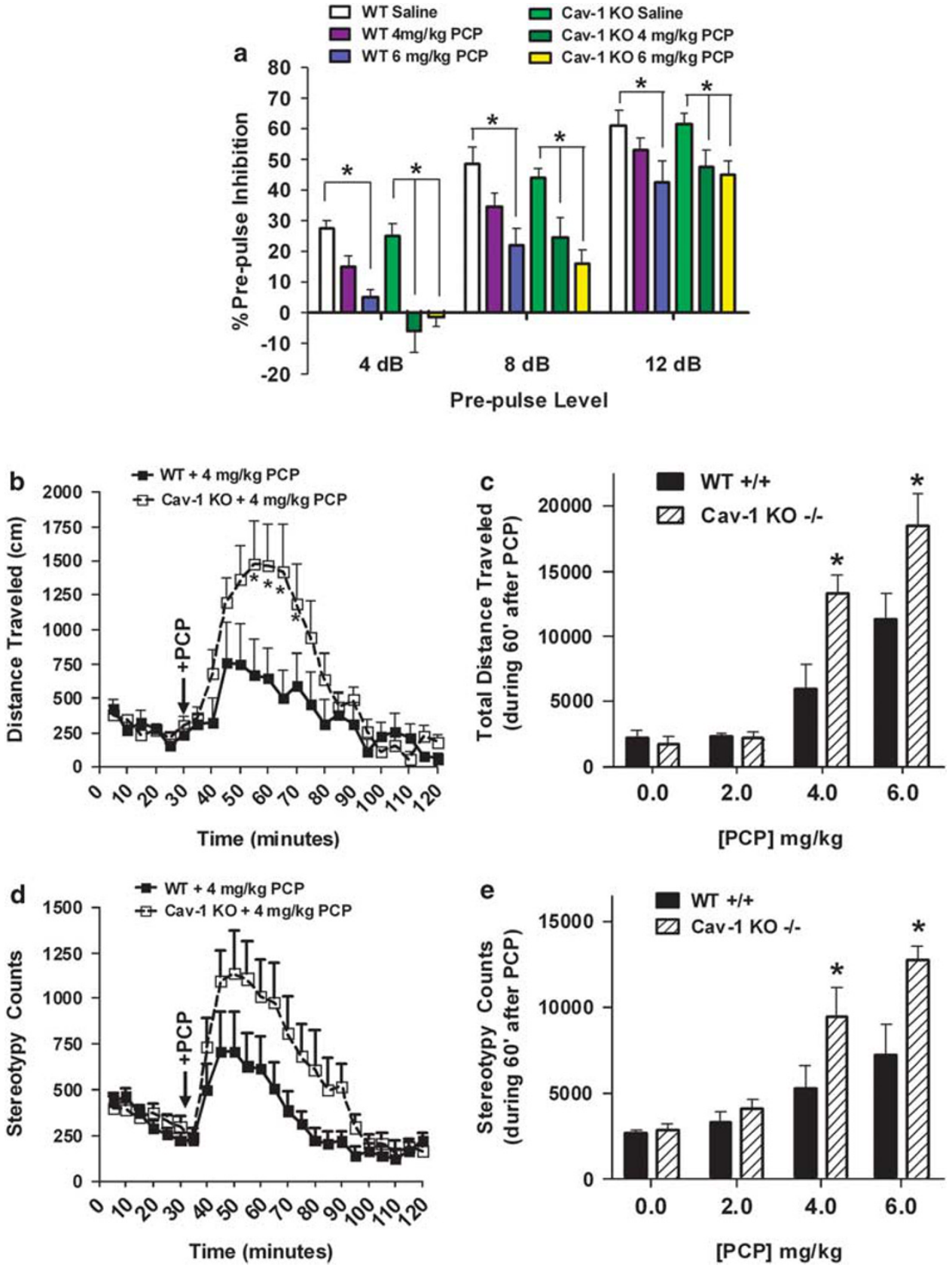

Figure 1 Genetic deletion of caveolin-1 (Cav-1) sensitizes mice to the behavioral effects of the psychotomimetic phencyclidine (PCP). (a) Cav-1 knockout (KO) mice exhibit increased sensitivity to PCP disruption of sensory motor gaiting. Littermate wild-type (WT) and Cav-1 KO mice were injected with saline, 4 or $6 \mathrm{mg} \mathrm{kg}^{-1}$ PCP and immediately placed into acoustic startle response chambers. In all, $6 \mathrm{mg} \mathrm{kg}^{-1} \mathrm{PCP}$ significantly decreased (disrupted) prepulse inhibition (PPI) at all prepulse levels in WT and Cav-1 KO mice. However, a lower dose of $4 \mathrm{mg} \mathrm{kg}^{-1} \mathrm{PCP}$ significantly disrupted PPI in only Cav-1 KO but not WT mice (mean \pm s.e.m., $n=8$ to 12 littermate pairs; ${ }^{*} P<0.05$ versus WT saline or Cav-1 KO saline at each prepulse level). (b, c) PCP-induced hyperlocomotion is increased in Cav-1 KO mice. Littermate WT and Cav-1 KO mice were placed into open field locomotion chambers and allowed to acclimate; $30 \mathrm{~min}$ later mice were injected with PCP and the total distance traveled (cm) was determined. Cav-1 KO mice exhibited a greater sensitivity to $4 \mathrm{mg} \mathrm{kg}^{-1} \mathrm{PCP}$ than WT mice (panel b, mean \pm s.e.m., $n=9$ littermate pairs; ${ }^{*} P<0.05$ versus WT mice at time indicated). Cav-1 KO mice also displayed increased total distance traveled in response to 4 and $6 \mathrm{mg} \mathrm{kg}^{-1}$ PCP compared with WT mice (panel c, mean \pm s.e.m., $n=8$ to 12 littermate pairs; ${ }^{\star} P<0.05$ versus WT mice in each group). (d, e) Stereotypy movement is increased in Cav-1 KO mice in response to $4 \mathrm{mg} \mathrm{kg}^{-1} \mathrm{PCP}$ (panel d, mean \pm s.e.m., $n=9$ littermate pairs). Cav-1 KO mice displayed increased total stereotypy counts in response to 4 and $6 \mathrm{mg} \mathrm{kg}^{-1}$ PCP compared with WT mice (panel e, mean \pm s.e.m., $n=8$ to 12 littermate pairs; ${ }^{*} P<0.05$ versus WT mice in each group).

points (Figure 3a). In a dose response trial of DOI (Figure $3 b$ ), total mouse head twitches were attenuated in Cav-1 KO mice at concentrations above $1.0 \mathrm{mg} \mathrm{kg}^{-1}$, further indicating that genetic loss of Cav-1 attenuates agonist activity at $5-\mathrm{HT}_{2 \mathrm{~A}}$ receptors. ${ }^{125} \mathrm{I}-\mathrm{DO}$ receptor autoradiography (Supplementary Figure $\mathrm{S} 3$ ) and radioligand binding (Supplementary Figure S4) in frontal cortex revealed no difference in $5-\mathrm{HT}_{2 \mathrm{~A}}$ receptor-binding sites between the 

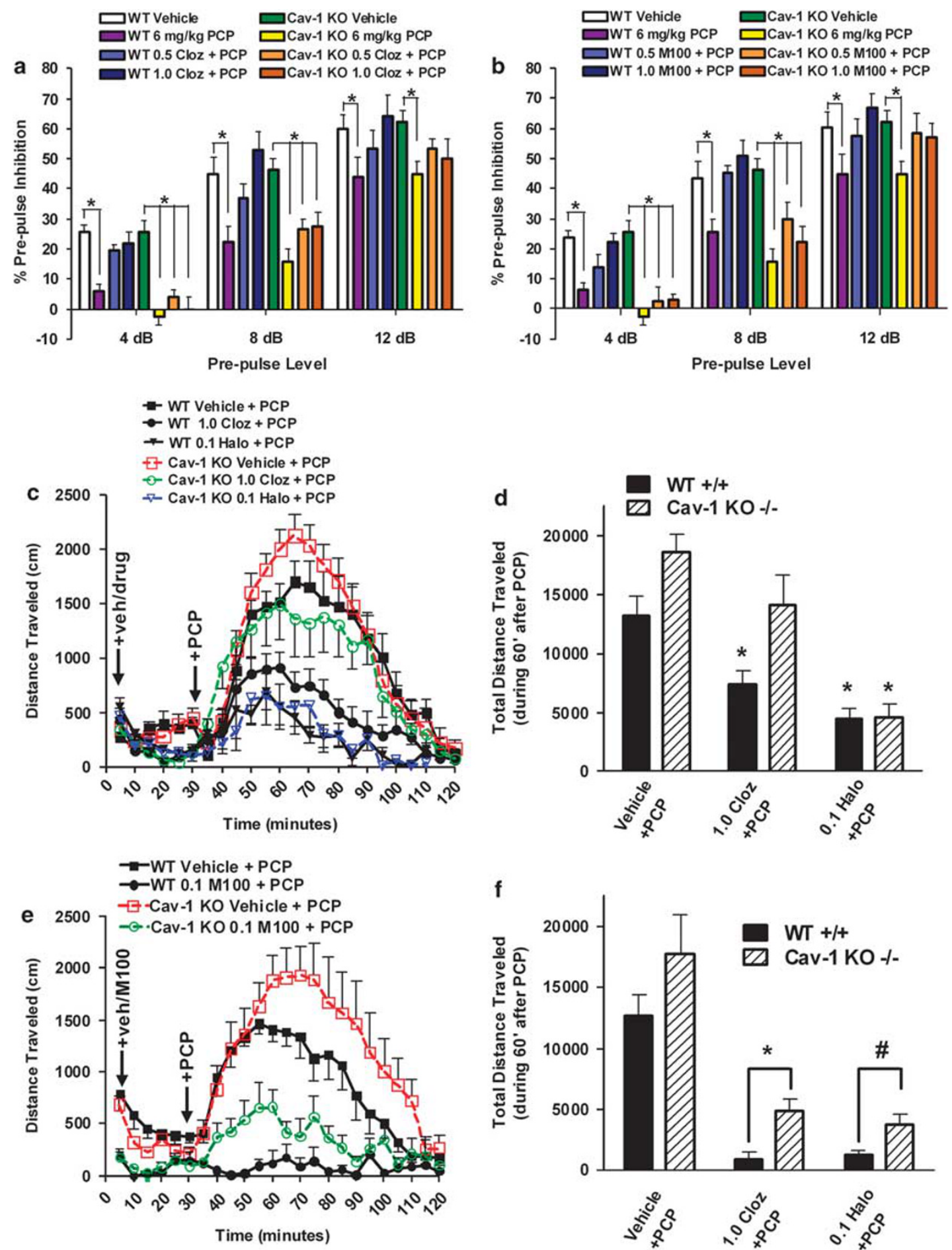

Figure 2 Caveolin-1 (Cav-1) knockout (KO) attenuates atypical antipsychotic and 5- $\mathrm{HT}_{2 \mathrm{~A}}$ antagonist drug actions in the phencyclidine (PCP) mouse model of psychosis. (a, b) Cav-1 KO renders clozapine and M100907 less effective at normalizing disrupted sensory motor gaiting. Littermate wild-type (WT) and Cav-1 KO mice received injections of vehicle, vehicle $+6 \mathrm{mg} \mathrm{kg}^{-1} \mathrm{PCP}$, clozapine $\left(0.5\right.$ or $\left.1.0 \mathrm{mg} \mathrm{kg}^{-1}\right)+\mathrm{PCP}$ or M100907 $\left(0.5\right.$ or $\left.1.0 \mathrm{mg} \mathrm{kg}^{-1}\right)+\mathrm{PCP}$ followed by measurement of acoustic startle responses. $6 \mathrm{mg} \mathrm{kg}^{-1}$ PCP significantly decreased (disrupted) prepulse inhibition (PPI) in both genotypes at all prepulse levels. Clozapine normalized the disrupted PPI to near vehicle levels in WT mice; however, clozapine was ineffective at normalizing PCP disrupted PPI in Cav-1 KO mice at 4 and $8 \mathrm{~dB}$ prepulse levels (panel a, mean \pm s.e.m., $n=12$ littermate pairs; ${ }^{*} P<0.05$ versus vehicle-treated WT or vehicle-treated Cav-1 KO mice). Similarly, M100907 normalized the disrupted PPI to near vehicle levels in WT mice but this was prevented in Cav-1 KO mice at 4 and $8 \mathrm{~dB}$ prepulse levels (panel b, mean \pm s.e.m., $n=10$ littermate pairs, ${ }^{\star} P<0.05$ versus vehicle-treated WT or vehicletreated Cav-1 KO mice). (c-f) Clozapine and M100907, but not haloperidol, are less effective at normalizing PCP-induced hyperlocomotion in Cav-1 KO mice. Clozapine treatments significantly decreased the total distance traveled after PCP in WT but not Cav-1 KO mice; however, haloperidol was equally effective in both genotypes (panels $c$ and d, mean \pm s.e.m., $n=8$ littermate pairs; ${ }^{*} P<0.05$ versus vehicle WT mice or vehicle Cav- $1 \mathrm{KO}$ mice). M100907 was also less effective at normalizing PCP-induced hyperlocomotion in Cav-1 KO mice compared with the drug activity in WT animals (panels e and f; mean \pm s.e.m., $n=8$ littermate pairs; ${ }^{*} P<0.05$ versus WT $0.1 \mathrm{M} 100+$ PCP; ${ }^{\#} P<0.05$ versus WT M100 + PCP). 

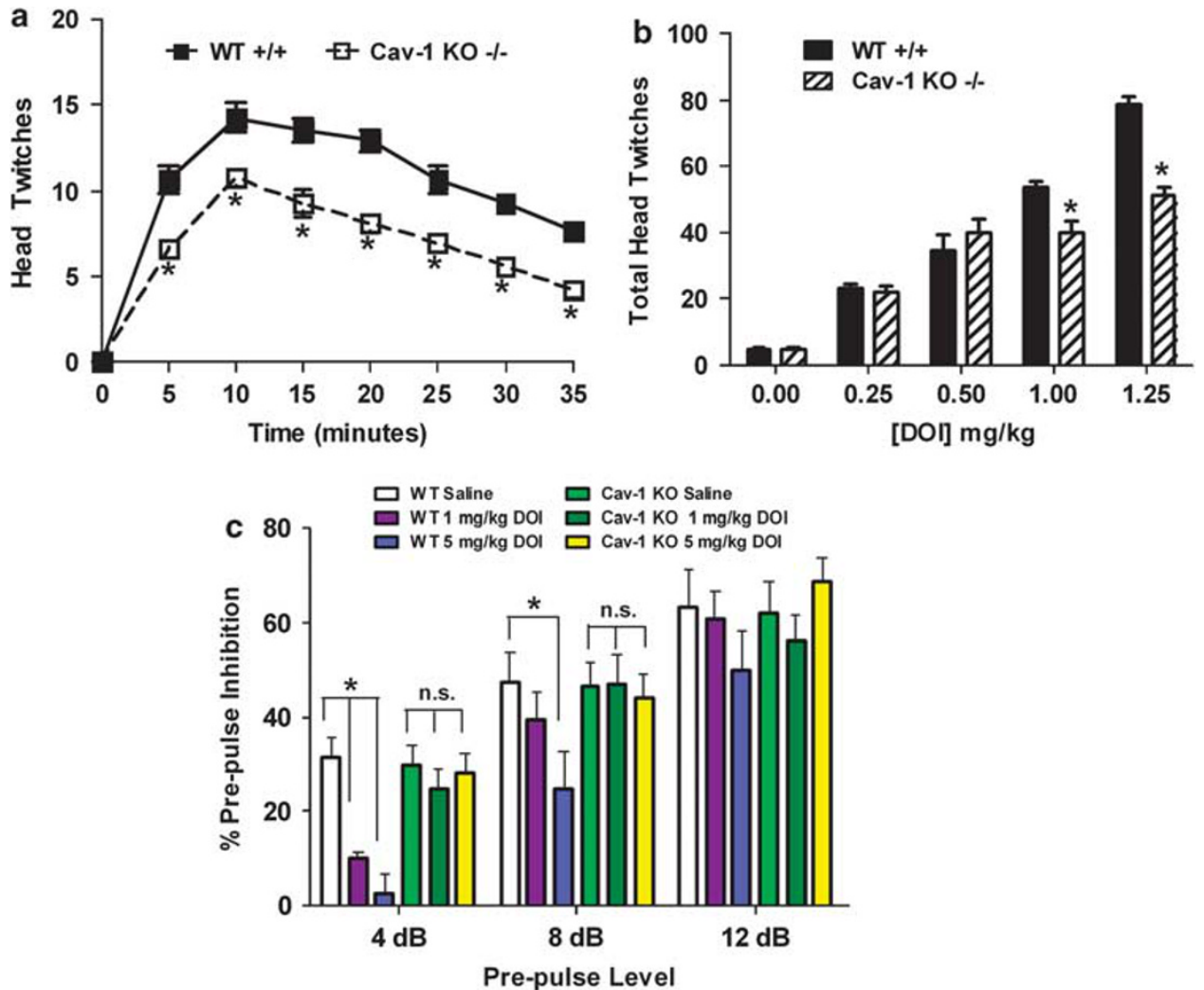

Figure 3 Mouse behavioral responses to the 5- $\mathrm{HT}_{2 \mathrm{~A}}$ agonist 2,5-dimethoxy-4-iodoamphetamine (DOI) are attenuated by genetic deletion of caveolin-1 (Cav-1). (a) Mouse head twitch responses to the $5-\mathrm{HT}_{2 \mathrm{~A}}$ agonist DOI are decreased by Cav-1 knockout (KO). Littermate wild-type (WT) and Cav-1 KO mice were injected with $1.25 \mathrm{mg} \mathrm{kg}^{-1} \mathrm{DOI}$ and head twitches scored over a 35 -min period; DOI-induced head twitches were attenuated in Cav-1 KO mice at all-time points (panel a, mean \pm s.e.m., $n=12$ littermate pairs; ${ }^{*} P<0.05$ versus WT mice). (b) Dose responses of DOI on total mouse head twitches over a 35-min period are shown; head twitches were attenuated in Cav-1 KO mice only at higher concentrations (panel b, mean \pm s.e.m., $n=6$ to 8 littermate pairs; ${ }^{*} P<0.05$ versus WT mice at each concentration). (c) DOI disruption of sensory motor gaiting in mice is prevented by Cav-1 KO. Littermate WT and Cav-1 KO mice were injected with saline, 1 or $5 \mathrm{mg} \mathrm{kg}^{-1} \mathrm{DOI}$ and 10 min later placed into acoustic startle response chambers to assess prepulse inhibition (PPI). DOI significantly decreased (disrupted) PPI at 4 and $8 \mathrm{~dB}$ prepulse levels in WT but not Cav-1 KO mice (panel c, mean \pm s.e.m., $n=8$ littermate pairs; ${ }^{*} P<0.05$ versus WT saline-treated mice at each prepulse level). NS, nonsignificant.

genotypes. Therefore, although a reduction in atypical antipsychotic drug action as well as the behavioral responses to $\mathrm{DOI}$ are seen in Cav-1 KO mice, these are not due to detectable changes in the overall number of cortical $5-\mathrm{HT}_{2 \mathrm{~A}}$ receptors, but rather could be due to a decrement in the signaling function of the receptors.

Given the aforementioned findings, we next determined whether Cav-1 might directly modulate $5-\mathrm{HT}_{2 \mathrm{~A}}$ signaling. $5-\mathrm{HT}_{2 \mathrm{~A}}$ agonists such as $\mathrm{DOI}$ are known to selectively induce the expression of the immediate early response genes c-Fos and egr-1 in the mouse cortex. ${ }^{16,29}$ In WT animals, DOI significantly increased cortical c-Fos and egr-1 mRNA expression. Notably, DOI-treated Cav-1 KO mice exhibited no significant induction of $c$-Fos or egr-1, indicating genetic loss of Cav-1 attenuates signaling activity at 5- $\mathrm{HT}_{2 \mathrm{~A}}$ receptors in the mouse cortex in vivo (Figures $4 \mathrm{a}$ and $\mathrm{b}$ ). In comparison, animals heterozygous for the Cav-1 gene exhibited a reduced c-Fos induction in response to DOI, suggesting a gene dosage effect. As 5- $\mathrm{HT}_{2 \mathrm{~A}}$ receptors couple principally to $\mathrm{G} \alpha \mathrm{q}$ and on ligand activation, signal via the $\mathrm{G} \alpha \mathrm{q} / \mathrm{phospholipase}$ $\mathrm{C} \beta / \mathrm{IP}_{3}$ pathway to mobilize calcium release from intracellular stores, we tested if Cav-1 altered $5-\mathrm{HT}_{2 \mathrm{~A}}$-induced calcium signaling in primary cortical neurons isolated from WT and Cav-1 KO embryonic mice. As previously detailed, ${ }^{30} \mathrm{DOI}$ does not elicit a detectable calcium response in primary neurons probably due to a low expression of endogenous 5- $\mathrm{HT}_{2 \mathrm{~A}}$ receptors; however, WT neurons expressing virally transduced $5-\mathrm{HT}_{2 \mathrm{~A}}$ receptors exhibit a robust increase in intracellular calcium dye fluorescence after addition of DOI (Figure 4c). By contrast, Cav-1 KO neurons normalized for expression of equivalent $5-\mathrm{HT}_{2 \mathrm{~A}}$ receptor levels show attenuated responses. Quantification of fluorescence in individual cells determined the peak calcium response after DOI (Emax) as well as the total calcium response (area under curve) was significantly decreased by Cav-1 KO (Figure 4d) (WT cells peak calcium Emax $=0.72 \pm 0.04$, Cav- $1 \mathrm{KO}$ cells peak calcium $E$ max $=0.57 \pm 0.4^{*}$; WT cells total calcium response $=36.7 \pm 1.2$, Cav- $1 \mathrm{KO}$ cells total calcium response $=24.9 \pm 1.6^{\star} ;{ }^{\star} P<0.05$ versus WT cells). Taken together, these results indicate genetic loss of Cav-1 attenuates neuronal $5-\mathrm{HT}_{2 \mathrm{~A}}$ signaling in vitro and in vivo.

\section{Discussion}

The major finding of this study is that genetic deletion of CAV1-a rare structural variant associated with schizophrenia-leads to an enhanced susceptibility to the psychotomimetic actions of PCP and reduced sensitivity to atypical but 

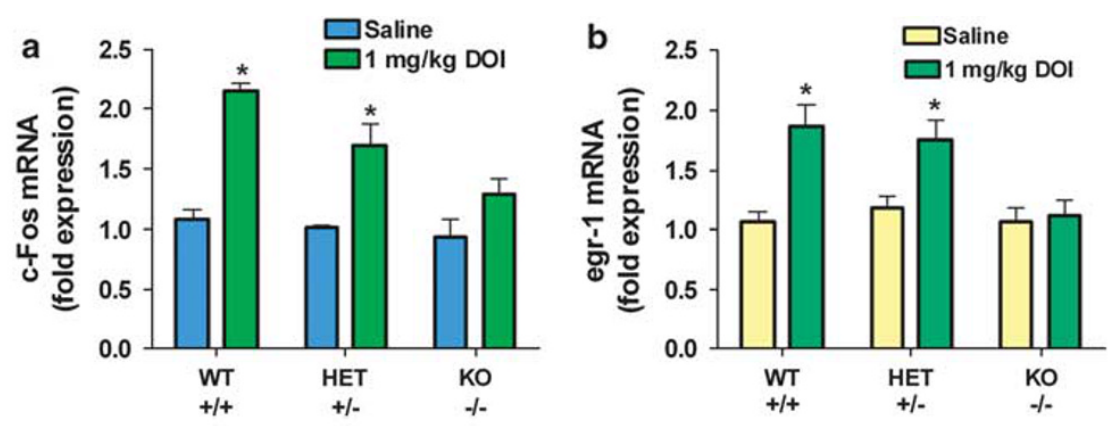
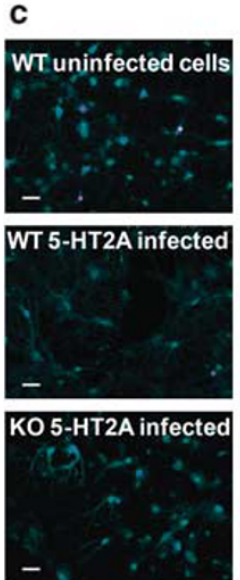
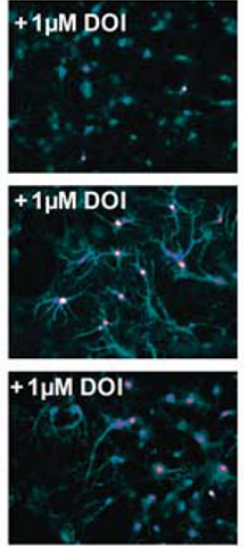
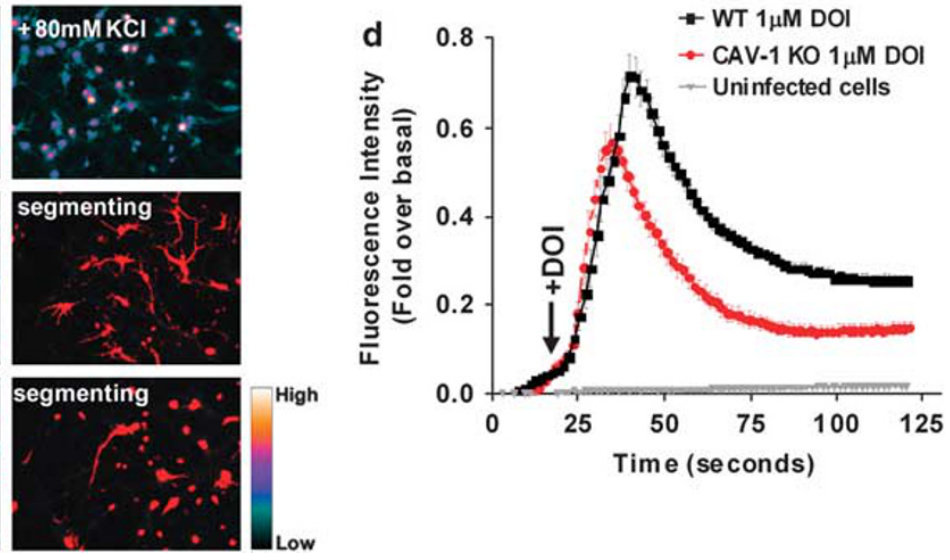

Figure $45-\mathrm{HT}_{2 \mathrm{~A}}$ signaling responses to the agonist 2,5-dimethoxy-4-iodoamphetamine (DOI) are attenuated in vivo and in vitro by genetic deletion of caveolin-1. (a, b) Littermate wild-type (WT) and caveolin-1 (Cav-1) knockout (KO) mice were injected with $1.0 \mathrm{mg} \mathrm{kg}^{-1} \mathrm{DOI}, 1 \mathrm{~h}$ later frontal cortex was isolated, mRNA extracted and transcript levels of c-Fos and egr-1 determined by real-time PCR. DOI-induced c-Fos and egr-1 gene expression in frontal cortex was decreased in mice heterozygous for Cav-1; DOIinduced gene expression was prevented in $\mathrm{KO}$ mice (panels a and $\mathrm{b}$, mean \pm s.e. $\mathrm{m}$., $n=5$ littermate mice for each group; ${ }^{*} P<0.05$ versus saline-treated mice in each group). (c) Neuronal $5-\mathrm{HT}_{2 \mathrm{~A}}$ receptor calcium signaling is decreased by genetic deletion of Cav-1. Primary cortical neurons were isolated from WT and Cav-1 KO mice and cultured in 96-well plates. Neurons were infected with lentiviruses encoding $5-\mathrm{HT}_{2 \mathrm{~A}}$ receptors and intracellular calcium release was determined by fluorescence calcium dye imaging using high-content imaging microscopy. Images of cells are shown before (left panels) and after (middle panels) an automated addition of the 5- $\mathrm{HT}_{2 \mathrm{~A}}$ agonist $\mathrm{DOI}$. WT neurons expressing $5-\mathrm{HT}_{2 \mathrm{~A}}$ receptors (middle panels) exhibited a robust increase in intracellular calcium dye fluorescence after DOI. In comparison, $\mathrm{KO}$ neurons expressing $5-\mathrm{HT}_{2 \mathrm{~A}}$ receptors (lower panels) displayed a reduced intracellular calcium dye fluorescence after agonist. The calcium dye fluorescence in individual responding cells in the wells (segmenting) was determined. (d) Quantification of calcium dye fluorescence in individual cells is shown and expressed as fold over initial fluorescence; KO neurons exhibited a reduced peak and total calcium response (Figure 4 d, mean fluorescence intensity \pm s.e.m. from $>90$ cells per group from $n=3$ cell preparations).

not typical antipsychotic drugs. This study also provides the first in vivo evidence that the scaffolding protein Cav-1 influences both central nervous system receptor function and psychoactive drug activity.

Scaffolding-related control mechanisms are now clearly important for the function of many receptors and ion channels and scaffolding proteins in general have been implicated in schizophrenia as well as many other neuropsychiatric diseases. ${ }^{31-33}$ Thus, for example, in the 22q11.2 microdeletions arising in DiGeorge syndrome, which results in cognitive deficits and a high risk of developing schizophrenia, the protein ZDHHC8 has been identified as a palmitoyltransferase for PSD95 that controls PSD95 function and its ability to scaffold proteins in the postsynaptic density. ${ }^{34}$ Genetic deletion of ZDHHC8 in DiGeorge syndrome may therefore disrupt synaptic glutamatergic and G protein-coupled receptor (GPCR) scaffolding with PSD95 and dysregulate receptor functions contributing to pathologies associated with schizophrenia. ${ }^{16}$ Here, we show that disruptions of Cav-1, an important neuronal signaling scaffold, may contribute to the mechanisms causing schizophrenia and possibly other neuropsychiatric disorders.
Our identification here of Cav-1-mediated regulation of $5-\mathrm{HT}_{2 \mathrm{~A}}$ receptors raises the intriguing possibility for altered $5-\mathrm{HT}_{2 \mathrm{~A}}$ receptor function in individuals with schizophrenia that possess the CAV1 mutation. The observation that Cav-1 KO mice exhibit increased sensitivity to the psychotomimetic effects of PCP (Figure 1), a phenomenon also observed in humans with schizophrenia, ${ }^{20,21}$ is a compelling behavioral observation bridging the mouse model and human schizophrenia. The mechanisms underlying this enhanced PCP sensitivity in Cav-1 KO mice are unknown, although our findings implicate dysfunctional serotonergic signaling. Recent studies have also indicated altered mGluR function in Cav-1 KO mice as well as alterations in synaptic plasticity. ${ }^{11,35}$ NMDA receptors are not currently known to directly interact with caveolins, but both proteins do colocalize in hippocampal neurons where Cav-1 is required for NMDA receptor signaling. ${ }^{36}$ However, hippocampal NMDA-mediated long-term depression is not altered in Cav-1 KO mice, although the mGluR-mediated long-term depression is altered. ${ }^{35}$ Therefore, this enhanced response to $\mathrm{PCP}$ observed in Cav-1 KO mice may involve changes in 
mGluR-NMDA receptor function, or, possibly, altered NMDA receptor scaffolding/trafficking by lack of Cav-1, both possibilities warrant future study.

Caveolins are thought to regulate GPCR signaling cascades by two distinct mechanisms (1) Caveolin scaffolding of GPCRs, G proteins and their associated effectors in membrane microdomains to regulate protein-protein interactions and therefore signal transduction and, (2) caveolin regulation of receptor trafficking as caveolins can control a distinct form of clathrin-independent endocytosis. ${ }^{9,10}$ However, exactly how caveolins function in the central nervous system remains unclear, although recent evidence indicates Cav-1 controls constitutive receptor endocytosis in hippocampal neurons for some GPCRs such as the mGluR $_{1 / 5}{ }^{11}$ and signaling by hippocampal mGluR $_{1 / 5}$ receptors to control hippocampal long-term depression is abnormal in Cav-1 KO mice. ${ }^{35}$ This is particularly interesting as both mGluR $_{1 / 5}$ and 5$\mathrm{HT}_{2 \mathrm{~A}}$ receptors couple primarily through $\mathrm{G} \alpha \mathrm{q}$ mechanisms, suggesting multiple GPCR-G $\alpha$ q signaling cascades may require Cav-1. Consistent with this notion, in our previous investigations, we determined that Cav-1 and Cav-2 interact with $5-\mathrm{HT}_{2 \mathrm{~A}}$ receptors and stable knockdown of caveolins profoundly inhibits G $\alpha q$-mediated calcium signaling. ${ }^{14} \mathrm{We}$ also previously determined that the $\mathrm{Cav} / 5-\mathrm{HT}_{2 \mathrm{~A}}$ interaction promotes a complex between $\mathrm{G} \alpha q$ and the receptor and this may be a mechanism by which caveolin influences $5-\mathrm{HT}_{2 \mathrm{~A}}$ receptor signaling and pharmacology.

Cav-1 scaffolding also seems essential for the salutary actions of atypical antipsychotic drugs in mice. Using the PCP mouse model of psychosis, we determined that genetic loss of Cav-1 attenuated the ability of the atypical antipsychotics clozapine and olanzapine to normalize the PCP-disrupted $\mathrm{PPI}$ and the PCP-induced hyperlocomotion (Figure 2). Cav-1 $\mathrm{KO}$ similarly attenuated the activity of the selective $5-\mathrm{HT}_{2 \mathrm{~A}}$ antagonist M100907 in both behavioral tests. However, the typical antipsychotic haloperidol, which acts primarily as a $D_{2}$-dopamine receptor antagonist, was equally effective in WT and Cav-1 KO mice. In addition, the increased potency of PCP in Cav-1 KO mice (Figure 1) cannot thoroughly explain the reduced efficacy of these atypical antagonists in behavioral studies. This is because the relative difference in PCP alone effects between WT and Cav-1 KO mice is not proportional to those differences observed between WT and Cav-1 KO mice after treatment with PCP and antipsychotics (Figure 2). These results indicate atypical antipsychotics, acting via antagonism at $5-\mathrm{HT}_{2 \mathrm{~A}}$ and other receptors, require the expression of Cav-1 for their full efficacy in mice. As total receptor number is unchanged by Cav- $1 \mathrm{KO}$, this requirement for Cav-1 could be explained by an attenuated $5-\mathrm{HT}_{2 \mathrm{~A}}$ receptor signaling. We recently determined that the presynaptic component of the serotonergic neuronal system is required for clozapine's antipsychotic activity (that is, normal $5-\mathrm{HT}_{2 \mathrm{~A}}$ signaling via serotonin must be intact for effective antagonism by clozapine in these PCP behavioral studies). ${ }^{15}$ Therefore, the reduced $5-\mathrm{HT}_{2 \mathrm{~A}}$ signaling evident in Cav-1 $\mathrm{KO}$ mouse cortex (Figure 4a) in cultured cortical neurons (Figure 4b) and in behavioral responses (Figure 3 ) is an indication that receptor activity is also reduced; this could make antagonism of the receptors less effective. Also consistent with this notion, we recently determined that PSD95 $\mathrm{KO}$ mice exhibit reduced agonist-induced $5-\mathrm{HT}_{2 \mathrm{~A}}$ signaling, which is associated with decreased clozapine activity in the $\mathrm{KO}$ mice. ${ }^{16}$ The findings here that Cav-1 is required for antipsychotic activity, along with our recent PSD95 $\mathrm{KO}$ mice results, indicate that $5-\mathrm{HT}_{2 \mathrm{~A}}$ signaling via scaffolding proteins appears to be a requirement for atypical antipsychotic activity in mouse models.

In summary, we have found that Cav-1 KO mice are supersensitive to the psychotomimetic actions of PCP. We also discovered that genetic loss of CAV1 attenuates the biochemical and behavioral actions of atypical antipsychotic drugs and that $5-\mathrm{HT}_{2 \mathrm{~A}}$ agonists and inverse agonists in vitro and in vivo have attenuated effects in Cav-1 KO mice. These findings provide fresh insights into the roles of caveolin in the central nervous system and provide support for the involvement of caveolin in the etiology of schizophrenia. Given that Cav-1 KO mice show resistance to the apparent antipsychotic actions of clozapine and olanzapine, it appears that caveolin is also required for atypical antipsychotic drug actions.

\section{Conflict of interest}

The authors declare no conflict of interest.

Acknowledgements. This work was supported by research grants from the National Institutes of Health, PHS RO1MH61887, U19MH82441, R01DA017204 and HHSN-271-2008-00025-C to BLR MF was supported by an NIH National Research Service Award F31MH091921-01. JAA was supported by NIH National Research Service Award training grant T32HD040127-07 and the UNC-Carolina Institute for Developmental Disabilities.

1. Purcell SM, Wray NR, Stone JL, Visscher PM, O'Donovan MC, Sullivan PF et al. Common polygenic variation contributes to risk of schizophrenia and bipolar disorder. Nature 2009; 460: 748-752.

2. Shi J, Levinson DF, Duan J, Sanders AR, Zheng Y, Pe'er I et al. Common variants on chromosome 6p22.1 are associated with schizophrenia. Nature 2009; 460: 753-757.

3. Stefansson H, Ophoff RA, Steinberg S, Andreassen OA, Cichon S, Rujescu D et al. Common variants conferring risk of schizophrenia. Nature 2009; 460: 744-747.

4. Stefansson H, Rujescu D, Cichon S, Pietilainen OP, Ingason A, Steinberg $S$ et al. Large recurrent microdeletions associated with schizophrenia. Nature 2008; 455: 232-236.

5. Jaaro-Peled H, Ayhan Y, Pletnikov MV, Sawa A. Review of pathological hallmarks of schizophrenia: comparison of genetic models with patients and nongenetic models. Schizophr Bull 2010; 36: 301-313.

6. Karayiorgou M, Simon TJ, Gogos JA. 22q11.2 microdeletions: linking DNA structural variation to brain dysfunction and schizophrenia. Nat Rev Neurosci 2010; 11: 402-416.

7. Walsh T, McClellan JM, McCarthy SE, Addington AM, Pierce SB, Cooper GM et al. Rare structural variants disrupt multiple genes in neurodevelopmental pathways in schizophrenia. Science 2008; 320: 539-543.

8. Cohen AW, Hnasko R, Schubert W, Lisanti MP. Role of caveolae and caveolins in health and disease. Physiol Rev 2004; 84: 1341-1379.

9. Ostrom RS. New determinants of receptor-effector coupling: trafficking and compartmentation in membrane microdomains. Mol Pharmacol 2002; 61: 473-476.

10. Allen JA, Halverson-Tamboli RA, Rasenick MM. Lipid raft microdomains and neurotransmitter signalling. Nat Rev Neurosci 2007; 8: 128-140.

11. Francesconi A, Kumari R, Zukin RS. Regulation of group I metabotropic glutamate receptor trafficking and signaling by the caveolar/lipid raft pathway. J Neurosci 2009; 29: 35903602.

12. Boulware MI, Kordasiewicz H, Mermelstein PG. Caveolin proteins are essential for distinct effects of membrane estrogen receptors in neurons. J Neurosci 2007; 27: 9941-9950.

13. Heiman M, Schaefer A, Gong S, Peterson JD, Day M, Ramsey KE et al. A translational profiling approach for the molecular characterization of CNS cell types. Cell 2008; 135: 738-748.

14. Bhatnagar A, Sheffler DJ, Kroeze WK, Compton-Toth B, Roth BL. Caveolin-1 interacts with 5-HT2A serotonin receptors and profoundly modulates the signaling of selected Galphaqcoupled protein receptors. J Biol Chem 2004; 279: 34614-34623.

15. Yadav PN, Abbas AI, Farrell MS, Setola V, Sciaky N, Huang XP et al. The presynaptic component of the serotonergic system is required for clozapine's efficacy. Neuropsychopharmacology 2011; 36: 638-651. 
16. Abbas Al, Yadav PN, Yao WD, Arbuckle Ml, Grant SG, Caron MG et al. PSD-95 is essential for hallucinogen and atypical antipsychotic drug actions at serotonin receptors. $J$ Neurosci 2009; 29: 7124-7136.

17. Farber NB. The NMDA receptor hypofunction model of psychosis. Ann NY Acad Sci 2003 1003: $119-130$

18. Geyer MA, Krebs-Thomson K, Braff DL, Swerdlow NR. Pharmacological studies of prepulse inhibition models of sensorimotor gating deficits in schizophrenia: a decade in review. Psychopharmacology (Berl) 2001; 156: 117-154

19. Moghaddam B. Bringing order to the glutamate chaos in schizophrenia. Neuron 2003; 40 881-884.

20. Luby ED, Cohen BD, Rosenbaum G, Gottlieb JS, Kelley R. Study of a new schizophrenomimetic drug; sernyl. AMA Arch Neurol Psychiatry 1959; 81: 363-369.

21. Lahti AC, Koffel B, LaPorte D, Tamminga CA. Subanesthetic doses of ketamine stimulate psychosis in schizophrenia. Neuropsychopharmacology 1995; 13: 9-19.

22. Razani B, Engelman JA, Wang XB, Schubert W, Zhang XL, Marks CB et al. Caveolin-1 null mice are viable but show evidence of hyperproliferative and vascular abnormalities. J Bio Chem 2001; 276: 38121-38138.

23. Linn GS, Javitt DC. Phencyclidine (PCP)-induced deficits of prepulse inhibition in monkeys. Neuroreport 2001; 12: 117-120.

24. Genedani S, Guidolin D, Leo G, Filaferro M, Torvinen M, Woods AS et al. Computerassisted image analysis of caveolin-1 involvement in the internalization process of adenosine A2A-dopamine D2 receptor heterodimers. J Mol Neurosci 2005; 26: 177-184.

25. Meltzer HY, Matsubara S, Lee JC. Classification of typical and atypical antipsychotic drugs on the basis of dopamine D-1, D-2 and serotonin2 pKi values. J Pharmacol Exp Ther 1989; 251: 238-246.

26. Roth BL, Sheffler DJ, Kroeze WK. Magic shotguns versus magic bullets: selectively non-selective drugs for mood disorders and schizophrenia. Nat Rev Drug Discov 2004; 3 353-359.

27. Keiser MJ, Setola V, Irwin JJ, Laggner C, Abbas Al, Hufeisen SJ et al. Predicting new molecular targets for known drugs. Nature 2009; 462: 175-181.

28. Glennon RA. Do classical hallucinogens act as 5-HT2 agonists or antagonists? Neuropsychopharmacology 1990; 3: 509-517.
29. Gonzalez-Maeso J, Yuen T, Ebersole BJ, Wurmbach E, Lira A, Zhou M et al. Transcriptome fingerprints distinguish hallucinogenic and nonhallucinogenic 5-hydroxytryptamine $2 \mathrm{~A}$ receptor agonist effects in mouse somatosensory cortex. J Neurosci 2003; 23: 8836-8843.

30. Strachan RT, Allen JA, Sheffler DJ, Roth BL. p90 Ribosomal S6 kinase 2, a novel GPCR kinase, is required for growth factor-mediated attenuation of GPCR signaling. Biochemistry 2010; 49: 2657-2671.

31. Owen MJ, Williams HJ, O'Donovan MC. Schizophrenia genetics: advancing on two fronts. Curr Opin Genet Dev 2009; 19: 266-270.

32. Fernandez E, Collins MO, Uren RT, Kopanitsa MV, Komiyama NH, Croning MD et al. Targeted tandem affinity purification of PSD-95 recovers core postsynaptic complexes and schizophrenia susceptibility proteins. Mol Syst Biol 2009; 5: 269.

33. Bayes A, van de Lagemaat LN, Collins MO, Croning MD, Whittle IR, Choudhary JS et al. Characterization of the proteome, diseases and evolution of the human postsynaptic density. Nat Neurosci 2011; 14: 19-21.

34. Mukai J, Dhilla A, Drew LJ, Stark KL, Cao L, MacDermott AB et al. Palmitoylationdependent neurodevelopmental deficits in a mouse model of $22 q 11$ microdeletion. Nat Neurosci 2008; 11: 1302-1310.

35. Takayasu Y, Takeuchi K, Kumari R, Bennett MV, Zukin RS, Francesconi A. Caveolin-1 knockout mice exhibit impaired induction of mGluR-dependent long-term depression at CA3-CA1 synapses. Proc Natl Acad Sci U S A 2010; 107: 21778-21783.

36. Head BP, Patel HH, Tsutsumi YM, Hu Y, Mejia T, Mora RC et al. Caveolin-1 expression is essential for $\mathrm{N}$-methyl-D-aspartate receptor-mediated $\mathrm{Src}$ and extracellular signalregulated kinase $1 / 2$ activation and protection of primary neurons from ischemic cell death. FASEB J 2008; 22: 828-840.

Translational Psychiatry is an open-access journal published by Nature Publishing Group. This work is licensed under the Creative Commons Attribution-NoncommercialNo Derivative Works 3.0 Unported License. To view a copy of this license, visit http://creativecommons.org/licenses/by-nc-nd/3.0/

\section{Supplementary Information accompanies the paper on the Translational Psychiatry website (http://www.nature.com/tp)}

